AN. MED INTERNA (Madrid) Vol. 18, N. ${ }^{\circ} 6$, pp. 319-322, 2001

\title{
Linfomatosis intravascular de presentación pulmonar
}

\author{
J.M. SASTRE, R. FOLGADO, O. BURGES*, Ma.D. ZARAGOZA, V. OLIVER \\ Servicio de Medicina Interna y *Departamento de Patología. Hospital Clínico. \\ Universidad de Valencia
}

INTRAVASCULAR LYMPHOMATOSIS PRESENTING IN THE LUNG

\begin{abstract}
RESUMEN
Se presenta un caso de linfomatosis intravascular cuyas primeras manifestaciones clínicas fueron síntomas pulmonares (tos no productiva, hipoxia y patrón radiológico reticulointersticial), seguidas de fiebre de origen desconocido y síndrome confusional. El diagnóstico se obtuvo mediante estudio histológico e inmunohistoquímico postmortem, observándose en el pulmón tabiques alveolares engrosados por la presencia de células atípicas en el interior de los capilares que también ocupaban las luces de pequeños vasos, evidenciándose de forma aislada salida de las mismas al estroma fibroso perivascular y peribronquial. Las técnicas inmunohistoquímicas sobre cortes en parafina revelaron en las células tumorales positividad para CD45 (Antígeno Leucocitario Común) y para CD20 (Marcador Pan-B), siendo negativas para CD45-Ro (Marcador Pan-T) y para el marcador endotelial CD34, inmunofenotipo de Linfoma Difuso Angiotropo de Células Grandes B, confirmándose por reordenación de cadenas pesadas de inmunoglobulinas (IgH). Se revisan las características principales de esta entidad, en especial sus manifestaciones pulmonares y la necesidad de incluirla en el diagnóstico diferencial procesos de apariencia clínica sistémica.
\end{abstract}

PALABRAS CLAVE: Linfomatosis intravascular. Pulmón. Linfoma.

\begin{abstract}
A case of intravascular lymphomatosis with predominant symptoms in the lung (cough and interstitial pulmonary infiltrates) is presented. Fever and confusion were lateness symptoms. Examination postmortem established the diagnosis. In the lung alveolar walls, small arterioles and the capillaries were occupied by atypical cells positives for CD45 and CD20, leukocyte and pan-B markers, and negatives for CD45-Ro and CD34, pan-T and endothelial markers. The main characteristics and the lung participation of this entity were reviewed.
\end{abstract}

KEY WORDS: Intravascular lymphomatosis. Lung. Lymphoma.

Sastre JM, Folgado R, Burges $O$, Zaragoza $M^{a} D$, Oliver V. Linfomatosis intravascular de presentación pulmonar. An Med Interna (Madrid) 2001; 18: 319-322.

\section{INTRODUCCION}

La linfomatosis intravascular (linfoma angiotrópico o angioendoteliosis maligna) es un trastorno linfoproliferativo maligno caracterizado por la proliferación de células mononucleares de carácter neoplásico en el interior de los pequeños vasos sanguíneos de prácticamente la totalidad del organismo, aunque muestra una mayor afinidad por el SNC y la piel. Descrita por primera vez en 1959 por Plfeger y Tappeiner, éstos autores propusieron que el origen de las células malignas sería el endotelio de los vasos afectados, y ello justificaría los distintos sinónimos empleados para denominarla, tales como angioendoteliomatosis proliferativa sistémica, angioendoteliomato- sis maligna o endoteliosis neoplásica (1). Trabajos recientes basados en estudios inmunohistoquímicos y en el análisis de Southern han demostrado que las células malignas tienen un origen linfoide, por lo que las denominaciones anteriores tienden a ser sustituidas por las de linfoma angiotrópico o linfomatosis intravascular (2-5). Se presenta generalmente como afectación multiorgánica de curso rápido y fatal, con pocos datos diagnósticos específicos, por lo que su diagnóstico es difícil, siendo en la mayoría de los casos postmortem (6-20).

Se comenta un caso de esta rara entidad que cursó fundamentalmente con síntomas pulmonares, fiebre de origen desconocido y síndrome confusional. El diagnóstico se obtuvo mediante estudio histológico e inmunohistoquímico postmor -

Trabajo aceptado: 11 de Noviembre de 1999

Correspondencia: Vicente Oliver. Servicio Medicina Interna. Hospital Clínico. Universidad de Valencia. Avda. Blasco Ibáñez, 17. 46010 Valencia. 
tem. El objetivo del presente trabajo reside en señalar las dificultades diagnósticas de esta entidad, haciendo hincapié en las poco frecuentes manifestaciones pulmonares y en la necesidad de incluirla en el diagnóstico diferencial de diversos procesos sistémicos.

\section{CASO APORTADO}

Mujer de 76 años, con antecedentes de talasemia minor, diabetes mellitus tipo 2 en tratamiento con insulina, y carcinoma basocelular en ala nasal tratado con radioterapia y posterior cirugía. Ingresó por presentar fiebre de una semana de evolución, acompañada de disnea de reposo, tos con expectoración, ortopnea y edemas periféricos, con $\mathrm{pO}_{2} 63 \mathrm{mmHg}\left(\mathrm{FiO}_{2} 21 \%\right), \mathrm{pCO}_{2} 38 \mathrm{mmHg}$, pH 7,41 y Rx de tórax normal. Con el diagnóstico de infección de vías respiratorias, se administraron antibióticos y oxígeno, mejorando clínica y analíticamente, y permitiendo alta hospitalaria. Reingresa 15 días después por fiebre, disnea de reposo y desorientación témporo-espacial, con disminución fluctuante del nivel de conciencia en ausencia de focalidad neurológica y de signos meníngeos. A la exploración, no presentaba lesiones cutáneas, adenopatías, masas ni hepatoesplenomegalia; la auscultación cardiaca fue normal, y en la auscultación respiratoria se detectaron crepitantes finos en ambas bases pulmonares. En la analítica realizada al ingreso destacaba: hipoxia severa sin retención de carbónico $\left(\mathrm{FiO}_{2} 21 \%, \mathrm{pO}_{2} 56 \mathrm{mmHg}, \mathrm{pCO}_{2} 38 \mathrm{mmHg}\right), \mathrm{pH} 7,35$, VSG $70 \mathrm{~mm}\left(1^{2} \mathrm{~h}\right), \mathrm{Hb} 7,7 \mathrm{~g} / \mathrm{dl}$, VCM 74 fl, LDH $426 \mathrm{U} / \mathrm{l}$, GOT $8 \mathrm{U} / 1$, GPT $6 \mathrm{U} / 1$, Fosfatasa alcalina $49 \mathrm{U} / \mathrm{l}$, creatinina $1 \mathrm{mg} / \mathrm{dl}$, ferritina $2196 \mathrm{ng} / \mathrm{ml}$, Factor reumatoide positivo y aumento de la proteína C reactiva, con ANA, anti-DNA, ANCA, e inmunocomplejos circulantes negativos. Estudio de BAAR en jugo gástrico negativo, así como la serología para infecciones respiratorias. Se realizó radiografía de tórax, punción lumbar y TAC cerebral, que resultaron normales. A los 5 días del ingreso en la radiografía de tórax se detectó patrón intersticial bilateral. Se realizaron nuevas pruebas diagnósticas siendo los resultados más relevantes: anemia microcítica con punteado basófilo y presencia de algunos esquistocitos, eliptocitos y dianocitos con trombopenia discreta, y presencia de células mononucleares anormales en sangre periférica (3\%). TAC tóraco-abdominal: demostró un patrón intersticial difuso bilateral con áreas parcheadas de condensación y derrame pleural bilateral así como hepatoesplenomegalia de carácter homogéneo. No se realizó fibrobroncoscopia diagnóstica dada la gravedad de la situación clínica de la paciente. Durante su ingreso presentó empeoramiento clínico, con fiebre elevada, estado de obnubilación sin focalidad neurológica y aparición de petequias y equimosis en ambos miembros superiores. La TAC cerebral fue normal. Hemocultivos fueron positivos para Enterococcus faecalis añadiéndose tratamiento antibiótico, pese a lo cual la evolución fue desfavorable, falleciendo a los dos meses de su ingreso.

Estudio postmortem. En la necropsia realizada a la paciente fueron pocas las lesiones macroscópicas encontradas: múltiples equimosis en piel de tórax y raíz de miembros superiores; enfisema pulmonar senil con antracosis, moderada arteriosclerosis aórtica, discreta esplenomegalia, cistitis petequial y diverticulosis colónica. El resto de órganos no mostraban alteraciones significativas. No se efectuó apertura de cráneo. Macroscópicamente no se identificaron adenopatías. Al estudio microscópico, destacaba la presencia en prácticamente todos los órganos estudiados, de una ocupación de las luces de los vasos arteriales y venosos de pequeño y mediano calibre por células redondeadas o poligonales, con núcleos grandes de contornos irregulares y cromatina grumosa, ocasionalmente con nucleolos prominentes, y un escaso citoplasma eosinófilo o anfófilo (Fig. 1). El parénquima pulmonar mostraba tabiques alveolares engrosados por la presencia de estas células atípicas en el interior de los capilares, que también ocupaban las luces de pequeños vasos (Fig. 2), evidenciándose de forma aislada salida de las mismas al estroma fibroso perivascular y peribronquial, y muy ocasionalmente

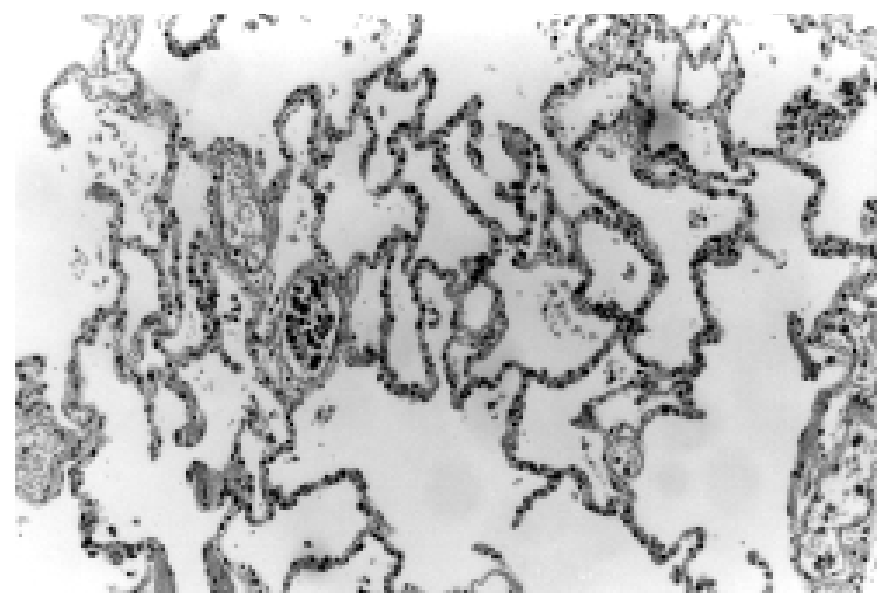

Fig. 1. Parénquima pulmonar en el que se evidencia centralmente un vaso con su luz ocupada por células tumorales. Solo de forma muy aislada se identificaron elementos linfoides atípicos en el interior de los alveolos. (Hematoxilina-Eosina x100).

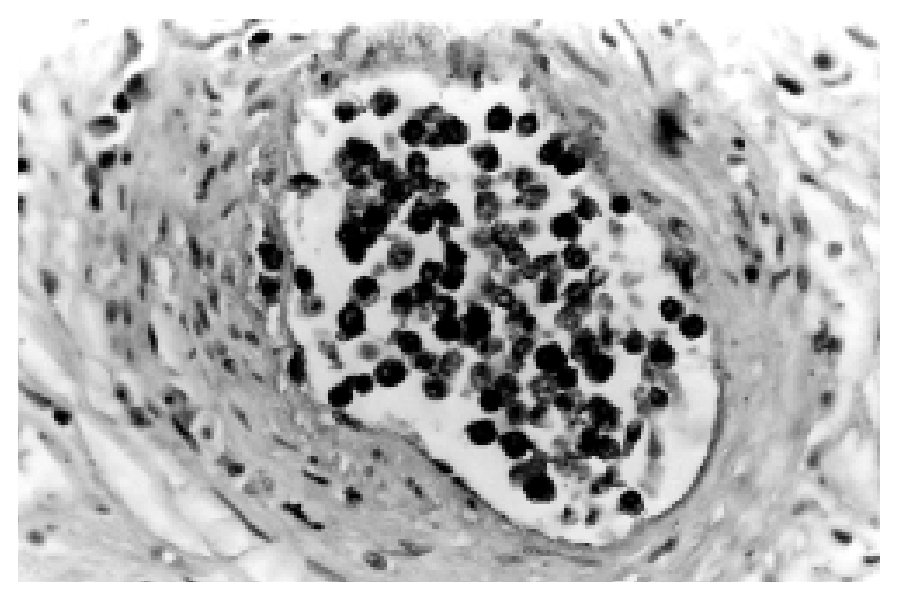

Fig. 2. Detalle de una luz vascular ocupada por células tumorales linfoides (Hematoxilina-Eosina x400).

al interior de algún alvéolo. Las técnicas inmunohistoquímicas sobre cortes en parafina del parénquima uterino revelaron en las células tumorales positividad para CD45 (Antígeno Leucocitario Común) y para CD20 (Marcador Pan-B), siendo negativas para CD45-Ro (Marcador Pan-T) y para el marcador endotelial CD34.

Las características morfológicas reseñadas, junto a este inmunofenotipo, permitieron tipificar la neoplasia como un Linfoma Difuso Angiotropo de Células Grandes Inmunofenotipo B, aspecto éste que se confirmó tras la demostración de reordenación de cadenas pesadas de inmunoglobulinas $(\mathrm{IgH})$ sobre tejido fijado en parafina.

\section{DISCUSIÓN}

En el presente caso la linfomatosis intravascular se manifestó inicialmente por síntomas y signos de afectación pulmonar, evolucionando posteriormente a la participación del Sistema Nervioso Central y piel. Durante la evolución se detectaron células anormales en sangre en escaso número, sin llegar a reconocerse como de origen linfoide y el factor reumatoide fue positivo. En la necropsia se demostró la diseminación intravascular con tan solo infiltración parenquimatosa extravascular a nivel uterino. El análisis mediante la técnica 
de Southern-Blot demostró que las células malignas eran de estirpe linfoide B, la más frecuente en estos linfomas.

La etiología de esta enfermedad permanece desconocida. Sólo en dos casos descritos en la literatura se han realizado estudios citogenéticos, y en ambos el único hallazgo común fue i(6p) (5,21-22), que es el segundo isocromosoma más común en los linfomas no Hodgkin, aunque no es específico de ningún subtipo en particular. La razón por la cual las células neoplásicas proliferan en el interior de los pequeños vasos sanguíneos y no infiltran las vísceras permanece desconocida. $\mathrm{Se}$ ha propuesto que diversas alteraciones en los receptores del "homing" presentes en la superficie de la célula linfocitaria y/o alteraciones moleculares en la superficie de la célula endotelial podrían ser responsables de la incapacidad de las células neoplásicas para extravasarse (23-26).

Aunque los órganos más comúnmente afectados son la piel y el SNC, otros muchos órganos pueden verse involucrados en esta enfermedad (corazón, pulmones, hígado, bazo, páncreas, riñones, glándulas adrenales, tracto genital, próstata, tiroides,...) (6-20,27-28). Por contra, la afectación de los ganglios linfáticos y de la médula ósea es poco común (9). Aunque la infiltración parenquimatosa es muy rara en esta enfermedad, en nuestro caso y tras la realización del estudio necrópsico, se observó la infiltración del estroma uterino por células atípicas, que en ningún momento se acompañó de clínica. A su vez se pudo observar en médula ósea celularidad linfoide atípica aislada, que no se constató en los estudios hematológicos realizados a partir de aspirados de médula ósea obtenidos in vivo. La sintomatología clínica es probablemente debida a la oclusión vascular y varía según su localización (8).

La revisión de 53 casos de linfomatosis intravascular descritos en la literatura (6-20,27-28) demuestra que los pacientes que desarrollan la enfermedad tienen una edad comprendida entre los 34 y los 87 años con una media de 70 años, sin que exista una clara predilección por un sexo. La clínica que aparece descrita con mayor frecuencia es la fiebre y la debida a la afectación del SNC y/o la piel (34 casos), seguida en orden de frecuencia por la secundaria a la afectación pulmonar ( 8 casos). Cuando la enfermedad se localiza en la piel se manifiesta en forma de nódulos subcutáneos múltiples de color rojizo o violáceo, equimosis o telangiectasias, y cuando lo hace en el SNC se manifiesta en forma de demencia progresiva subaguda, síndrome confusional o cuadros de focalidad muy diversos. También ha sido descrita la afectación de otros órganos manifestándose en 2 casos en forma de insuficiencia adrenal primaria (10); 4 casos con alteraciones hematológicas variadas como anemia hemolítica, microangiopatía trombótica, CID y/o síndrome hemofagocítico (14-15,17-18); y en un caso afectación nasal (16), síndrome nefrótico (29) o síndrome de secreción inadecuada de ADH (8).

En el presente caso, junto a la fiebre, las manifestaciones clínicas predominantes fueron secundarias a la afectación pul- monar, disnea, tos, hipoxemia e infiltración intersticial pulmonar; añadiéndose con posterioridad síndrome confusional y equimosis de localización preferente en miembros, datos típicos de la afectación del SNC y cutánea. Los casos de afectación pulmonar descritos en la literatura evidencian la presencia de disnea, tos no productiva e hipoxemia, junto a imágenes radiológicas de patrón reticulonodular difuso, sin signos de condensación ni cavitación (27-28). Un caso descrito en la literatura se acompaño de síndrome de secreción inadecuada de ADH.

Las alteraciones analíticas encontradas en la linfomatosis intravascular incluyen anemia, aumento de la VSG, alteraciones hidroelectrolíticas, hipoxemia y alteraciones de la función inmune con la positividad del factor reumatoide, la presencia de anticuerpos antinucleares o la presencia de anticuerpos dirigidos contra la superficie de los eritrocitos $(8-9,26)$. En nuestro caso destacaron la presencia de una anemia microangiopática y la positividad del factor reumatoideo. Ocasionalmente se han encontrado células anormales circulantes o en LCR (30-31). En nuestro caso, tan solo pudimos constatar aumentos variables de células grandes anormales en el hemograma, aunque en uno de los muchos hemogramas realizados encontramos la presencia de células grandes de aspecto reticulado; este hallazgo no se volvió a repetir en el resto de los hemogramas y frotis sanguíneos realizados. Los estudios del LCR fueron normales constatándose tan solo un aumento ligero de proteínas en el mismo.

La dificultad en el diagnóstico hace que en la mayor parte de los casos revisados este sólo pueda realizarse a través del estudio histológico e inmunohistoquímico postmortem; en los casos diagnosticados antemortem, el diagnóstico se obtuvo mediante biopsia de las lesiones cutáneas, del SNC o del tejido pulmonar. mediante fibrobroncoscopia o biopsia a cielo abierto. En nuestro caso la fibrobroncoscopia no pudo llevarse a cabo dada la mala situación clínica del paciente. En caso de sospecharse la enfermedad, la piel debería ser el primer lugar a biopsiar dada la frecuencia con que está infiltrada y su accesibilidad (20).

La linfomatosis intravascular tiene generalmente muy mal pronóstico. En los casos revisados la mortalidad se acerca al $80 \%$ con un rango de supervivencia que varía entre los 5 meses y los 9 años (media de 10-12 meses). En general la respuesta a la quimioterapia y/o radioterapia es escasa, pero en algunos casos se ha documentado una respuesta favorable con remisiones completas duraderas (31).

En conclusión, dado que el diagnóstico de la linfomatosis intravascular es difícil debido a la presencia de pocos datos específicos, esta enfermedad debería formar parte del diagnóstico diferencial de numerosas entidades clínicas entre las que destacarían los síndromes confusionales agudos, la demencia subaguda progresiva, las vasculitis, neoplasias ocultas y la fiebre de origen desconocido. La presentación pulmonar puede ser la primera manifestación de la enfermedad.

\section{Bibliografía}

1. Pfleger LM, Tappeiner J. Zur kenntis der sistemisierten endotheliomatose der cutanen blutgefäse (reticuloendotheliose) Hautart 1959; 10: 359-363.

2. Sheibani K, Battifora H, Winberg CD, Burke JS, Ben-Ezra J, Ellinger $\mathrm{GM}$ et al. Further evidence that "malignant angioendotheliomatosis" is an angiotropic large-cell lymphoma. N Engl J Med 1986; 314: 943-948.

3. Wick MR, Mills SE. Intravascular lymphomatosis: clinicopathologic features and differential diagnosis. Semin Diagn Pathol 1991; 8: 91101.

4. Lakhani SR, Hulman G, Hall JM, Slack DN, Sloane JP. Intravascular 
malignant lymphomatosis (angitropic large cell lymphma). A case report with evidence for $\mathrm{T}$-cell lineage with polymerase chain reaction analysis. Histopathology 1994; 25: 283-286.

5. Molina A, Lombard C, Donlon T, Bangs CD, Dorfman RF. Inmunohis tochemical and cytogenetic studies indicate that malignant angiendotheliomatosis is a primary intravascular (angiotropic) lymphoma. Cancer 1990; 66: 474-479.

6. Case Records of the Massachusetts General Hospital (case 31-1995). N Engl J Med 1995; 333: 992-9.

7. Case Records of the Massachusetts General Hospital (case 30-1996). N Engl J Med 1996; 335: 952-9.

8. Pellicone JT, Goldstein HB. Pulmonary Malignant Angioendotheliomatosis: presentation with fever and syndrome of inappropriate antidiuretic hormone. Chest 1990; 98: 1292-94.

9. Demirer T, Dail DH, Aboulafia DM. Four varied cases of intravascular lymphomatosis and a literature review. Cancer 1994; 73: 1738-1745.

10. Chu P, Costa J, Lachman MF. Angiotropic large cell lymphoma presenting as primary adrenal insufficiency. Hum Pathol 1996 ;27: 209-211.

11. Liszka U, Drlicek M, Hitzenberger P, Machacek E, Mayer H, Stockhammer G, Grisold W. Intravascular lymphomatosis: a clinicopathological study of three cases. J Cancer Res Clin Oncol 1994; 120: 164-168.

12. Glass J, Hochberg FH, Miller DC. Intravascular lymphomatosis: a systemic disease with neurologic manifestations. Cancer 1993; 71: 3156-62.

13. Stroup RM, Sheibani K, Moncada A, Purdy J, Battifora H. Angiotropic (intravascular) large cell lymphoma: a clinicopathologic study of seven cases with unique clinical presentations. Cancer 1990; 66: 1781-88.

14. Sill H, Höfler G, Kaufmann P, Horina J, Spuller E, Kleinert R, BehamSchimid C. Angiotropic large cell lymphoma presenting as thrombotic microangiopathy (thrombotic thrombocytopenic purpura). Cancer 1995; 75: $1167-1169$

15. Okada Y, Nakanishi I, Nomura H, Takeda R, Noromura A, Takekuma K. Angiotropic B-cell lymphoma with hemophagocytic syndrome. Path Res Pract 1994; 190: 718-724.

16. Wick MR, Banks PM, McDonald TM. Angioendotheliomatosis of the nose with fatal systemic dissemination. Cancer 1981; 48: 2510-2517.

17. Wick MR, Rocamora A. Reactive and malignant "angioendotheliomatosis": a discriminant clinicopathological study. J Cutan Pathol 1988; 15: $260-271$.

18. Stahl RL, Chan W, Duncan A, Corlley CC. Malignant angioendothelimatosis presenting as disseminated intravascular coagulopathy. Cancer
1991; 68: 2319-2323.

19. Arnn ET, Yam LT, Chi-Yang Li. Systemic angioendotheliomatosis presenting with hemolytic anemia. Am J Clin Pathol 1983; 80: 246-251.

20. Lafuente C, Dhimes-Tejada P, Rojo G, Carabias E. Linfomatosis intravascular, presentación de un caso y breve revisión de la entidad. An Med Interna (Madrid) 1995; 12: 243-245.

21. Domizio P, Hall PA, Cotter F, Amiel S, Tucker J, Besser GM, Levison A. Angiotropic large cell lymphoma: morphological, inmunohistochemical and genotypic studies with analysis of previous reports. Hematol Oncol 1989; 7: 195-206.

22. Bloomfield CD, Arthur DC, Frizzera G, Levine EG, Peterson BA, Gaj1peczalska KJ. Nonrandom chromosome abnormalities in lymphoma. Cancer Res 1983; 43: 2975-2984.

23. Gallatin WM, Weissman IL, Butcher EC. A cell-surface molecule involved in organ-especific homing o lymphocytes. Nature 1983; 304 : 30-34.

24. Ferry JA, Harris LA, Picker LJ, Weinberg DS, Rosales RK, Tapia J, Richardson EP. Intravascular lymphomatosis (malignant angioendotheliomatosis). A B-cell neoplasm expression surface homing receptors. Modern Pathol 1988; 1: 444-452.

25. Jalkanen S, Aho R, Kallajori M, Ekfors T, Nortamo P, Gahmberg C, Duijvestijn A, Kalimo H. Lymphocyte homing receptors and adhesion molecules in intravascular malignant lymphomatosis. Int $\mathrm{J}$ Cancer 1989; 44: 777-782.

26. Picker LJ, Medeiros LJ, Weiss LM, Warnke AR, Butcher EC. Expression of lymphocyte homing receptor antigen in non-Hodgkins lymphoma. Am J Pathol 1988; 130: 496-504.

27. Tan TB, Spaander PJ, Blaisse M, Gerritzen FM. Angiotropic large cell lymphoma presenting as intersticial lung disease. Thorax 1988; 43: 578579

28. Yousem SA, Colby TV. Intravascular lymphomatosis presenting in the lung. Cancer 1990; 65: 349-353.

29. Agar JWM, Gates PC, Vanghan SL, Machet D. Renal biopsy in angiotropic large cell lymphoma. Am J Kidney Dis. 1994;24:92-96.

30. Bhawan J, Wolff SM, Ucci AA, Bhan AK. Malignant lymphoma and malignant angiendotheliomatosis: one disease. Cancer 1985; 55: 570576

31. Di Giuseppe JA, Nelson WG, Seifter EJ, Boitnott JK, Mann RB. Intravascular lymphomatosis: a clinicopathologic study of 10 cases and assessment of response to chemotherapy. J Clin Oncol 1994; 12: 2573-79. 